\title{
HcrVf paralogs are present on linkage groups 1 and 6 of Malus
}

\author{
Giovanni A.L. Broggini, Paolo Galli, Gabriella Parravicini, Luca Gianfranceschi, \\ Cesare Gessler, and Andrea Patocchi
}

\begin{abstract}
Molecular markers derived from resistance gene analogs of $H c r V f$, the first apple resistance gene cloned, may pave the way to the cloning of additional apple scab resistance genes. The Malus $\times$ domestica 'Florina' (Vf) bacterial artificial chromosome (BAC) genomic library was screened by hybridization using HcrVf2 as a probe. Positive BAC clones were assembled into contigs and microsatellite markers developed from each contig mapped. Only linkage groups 1 and 6 contained $H c r V f 2$ paralogs. On linkage group 1, five loci in addition to the $V f$ locus were identified. A single locus was detected on linkage group 6. Representative BAC clones of these loci including the $V f$ locus were sequenced and the gene structure compiled. A total of 22 sequences, showing high sequence similarity to HcrVf2, were identified. Nine sequences were predicted to encode all seven protein domains described in HcrVf2, while three were truncated. Transcriptional analysis indicated that six genes with a complete HcrVf-like structure were constitutively expressed in young uninfected leaves of 'Florina'. The map position of each HcrVf analog was compared with the location of the major apple scab resistance genes. None of the major genes conferring scab resistance co-localized with HcrVf paralogs, indicating that they are unlikely to belong to the leucine-rich repeat - transmembrane class, which includes the $V f$ gene.
\end{abstract}

Key words: gene-for-gene, receptor-like protein, apple scab, Venturia inaequalis, fungal-plant relationships.

Résumé : Des marqueurs moléculaires dérivés d'analogues du gène de résistance $H c r V f 2$, le premier gène de résistance cloné chez le pommier, pourraient paver la voie en vue du clonage d'autres gènes de résistance à la tavelure du pommier. La banque génomique de clones BAC du Malus $\times$ domestica 'Florina' $(V f)$ a été criblée par hybridation en utilisant comme sonde HcrVf2. Des clones BAC positifs ont été assemblés en contigs, des marqueurs microsatellites ont été développés à partir de chaque contig et ces marqueurs ont été cartographiés. Seuls les groupes de liaison 1 et 6 portaient des paralogues de HcrVf2. Sur le groupe de liaison 1, cinq locus ont été identifiés en plus du locus $V f$ tandis qu'un seul locus a été identifié sur le groupe de liaison 6. Des clones BAC représentatifs de ces locus, incluant le locus $V f$, ont été séquencés et la structure des gènes a été déterminée. Au total, 22 séquences montrant une grande similarité avec la séquence de $H c r V f 2$ ont été identifiées. Il a été prédit que neuf des séquences codaient pour l'ensemble des sept domaines protéiques décrits

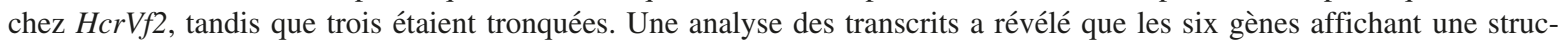
ture intacte de type HcrVf étaient exprimés constitutivement chez de jeunes feuilles non-infectées du 'Florina'. La position sur la carte génétique de chaque analogue $H c r V f$ a été comparée à l'emplacement des principaux gènes de résistance à la tavelure. Aucun des gènes majeurs conférant la résistance à la tavelure ne co-localisait avec les paralogues HcrVf, ce qui indique qu'il est peu vraisemblable qu'ils fassent partie de la classe des LRR-TM, laquelle inclut le gène $V f$.

Mots-clés : gène-pour-gène, protéine de type récepteur, tavelure du pommier, Venturia inaequalis, relations plantechampignon.

\section{Introduction}

Apple scab, caused by the ascomycetes Venturia inaequalis, is the most important fungal pathogen of the cultivated apple Malus $\times$ domestica (MacHardy 1996). The pathogen undergoes a gene-for-gene relationship with its host (Flor
1971). More than 14 apple resistance (R) genes conferring resistance to scab have been identified and the map position of the majority has been determined in the apple genome (Gessler et al. 2006). To date, a single apple scab R gene (HcrVf2 from the $V f$ region) that confers immunity to susceptible cultivars has been identified (Vinatzer et al. 2001;

Received 16 April 2008. Accepted 16 November 2008. Published on the NRC Research Press Web site at genome.nrc.ca on 21 January 2009.

Corresponding Editor: M. Francki.

G.A.L. Broggini, P. Galli, G. Parravicini, and C. Gessler. Plant Pathology, IBZ, ETH Zürich, Universitätstrasse 2, 8092 Zürich, Switzerland.

L. Gianfranceschi. Department of Biomolecular Sciences and Biotechnology, University of Milan, via Celoria 26, 20133 Milano, Italy. A. Patocchi. ${ }^{1}$ Phytopathology, Plant Protection, Agroscope-Changins-Wädenswil (ACW) Research Station, Schloss, P.O. 185, 8820 Wädenswil, Switzerland.

${ }^{1}$ Corresponding author (e-mail: andrea.patocchi@acw.admin.ch). 
Belfanti et al. 2004). The deduced amino acid sequence of $H c r V f 2$ shows high similarity to the $C f 9$ gene of the tomato, conferring resistance against Cladosporium fulvum, and is one of the best studied cases of gene-for-gene interactions characterized to date (Joosten and de Wit 1999). Similarly to $C f 9$, the $H c r V f 2$ protein structure has been subdivided into seven domains: a predicted signal peptide (domain A), the $\mathrm{NH}_{2}$ terminus of the mature protein (domain $\mathrm{B}$ ), an extracellular leucine-rich repeat (LRR) domain (domain C) possibly involved in recognition (Braun et al. 1991), domain $\mathrm{D}$ with an unknown function, an acidic domain (domain E), a hydrophobic domain thought to be the transmembrane region (domain F), and a basic domain (domain $\mathrm{G}$ ) representing the C terminus (Jones et al. 1994), anchoring the protein to the plasma membrane.

$\mathrm{R}$ gene analogs (RGA) associated with disease resistance are candidates for functional $\mathrm{R}$ genes. Among the several classes of $\mathrm{R}$ genes described in the literature, two contain nucleotide-binding site (NBS) motifs (Kruijt et al. 2005) and have been used to identify and map members of those RGA classes in apple (Baldi et al. 2004; Calenge et al. 2005) and other plant species (Leister et al. 1996; Collins et al. 1998; Shen et al. 1998). NBSs containing RGAs have been identified on all the linkage groups (LG) of apple, with the exception of LG13 and LG14. They are found in clusters and the largest cluster is located at the top of LG2. Several NBSRGAs were identified in regions where apple scab $\mathrm{R}$ genes were located or within the confidence interval of some scab resistance QTL (Baldi et al. 2004; Calenge et al. 2005; Gessler et al. 2006). Further studies by Gardiner et al. (2003) and Naik et al. (2006) detailed the mapping of additional RGAs in the apple genome. Naik et al. (2006) identified eight loci, belonging to different $\mathrm{R}$ gene classes, on LG2, LG5, LG11, LG12, and LG15. A different approach to map additional RGAs was adopted by Gardiner et al. (2003) and was based on the identification of apple EST sequences with high similarity to all five classes of $\mathrm{R}$ genes. Molecular markers were developed from ESTs tightly linked to $V f$ and to $\mathrm{Pl}_{2}$ and $P l_{M I S}$, two genes conferring resistance to powdery mildew. Although Gardiner et al. (2003) and Naik et al. (2006) were able to map RGA markers derived from $C f$-like EST sequences, the authors reported that it was not always possible to assign each EST unambiguously to a single class of R genes, since complete sequence information was not always available. Screening large-insert genomic libraries provides an alternative approach to identifying genes similar to known $\mathrm{R}$ genes (paralogs).

It has been shown that HcrVf genes are associated with apple scab resistance derived from Malus floribunda 821 (Belfanti et al. 2004; Malnoy et al. 2008). Therefore, it is possible that HcrVf paralogs may account for other apple scab resistances from sources other than M. floribunda 821 . This study reports on the identification and characterization of HcrVf2 paralogs in M. × domestica 'Florina', the development of SSR markers linked to these genes, and their genetic mapping within the apple genome. Furthermore, the expression of the HcrVf2 paralogs containing all of the predicted protein domains of $\mathrm{HcrVf2}$ was investigated in young uninoculated apple leaves. Finally, a phylogenetic study to elucidate the evolutionary pattern of the gene family was conducted on the identified paralogs.

\section{Materials and methods}

\section{Apple genomic library}

The genomic library consisted of 36864 bacterial artificial chromosome (BAC) clones generated from 'Florina' (Vinatzer et al. 1998). The library was previously used to perform the chromosome walking for the isolation of the $V f$ gene (Patocchi et al. 1999; Vinatzer et al. 2001). The library was spotted onto 96 nylon membranes (Hybond $\mathrm{N}+$ filters) as high-density arrays (1536 clones per filter).

\section{Southern blot hybridization with $\mathrm{HcrVf2}$}

The HcrVf2 probe, used for Southern blot hybridization, was amplified by PCR from BAC clone M18-4 (Vinatzer et al. 2001) using FD5for (5'-ATGGAGAGAACCATGAGAGTTG-3') and FD5rev (5'-TACTGGCATATTCGTCGCAG-3') primers, leading to the amplification of the complete open reading frame (ORF) of HcrVf2. The $50 \mu \mathrm{L}$ PCR consisted of $5 \mathrm{ng}$ of plasmid DNA, 3.5 units of Taq DNA polymerase (New England Biolabs, USA), $1 \times$ PCR buffer $(50 \mathrm{mmol} / \mathrm{L} \mathrm{KCl}, 1.5 \mathrm{mmol} / \mathrm{L} \mathrm{MgCl}$, and $10 \mathrm{mmol} / \mathrm{L}$ Tris- $\mathrm{HCl}$ ), $0.1 \mathrm{mmol} / \mathrm{L} \mathrm{dNTPs}$, and $0.3 \mu \mathrm{mol} / \mathrm{L}$ each of primers FD5for and FD5rev and was performed in a Perkin Elmer Cetus Gene Amp PCR System 9600 (Perkin Elmer, USA). The PCR amplification profile consisted of 35 cycles with each cycle having thermal conditions of $30 \mathrm{~s}$ at $94{ }^{\circ} \mathrm{C}$, $30 \mathrm{~s}$ at $60{ }^{\circ} \mathrm{C}$, and $2 \mathrm{~min}$ at $72{ }^{\circ} \mathrm{C}$. The PCR products were purified by excision from a $1 \%$ agarose gel $(0.5 \times \mathrm{TBE})$ using a WIZARD SV Clean-Up System Kit (Promega, USA) following the manufacturer's instructions and finally eluted in $100 \mu \mathrm{L}$ of $\mathrm{ddH}_{2} \mathrm{O}$. The $H c r V f 2$ probe was labeled with a Nick Translation System (Invitrogen, USA) using $200 \mathrm{ng}$ of the purified DNA and $1.5 \mu \mathrm{L}$ of $\left[\alpha-{ }^{32} \mathrm{P}\right] \mathrm{dCTP}(15 \mu \mathrm{Ci}$ $(555 \mathrm{kBq}))(\mathrm{GE}$ Healthcare, USA). Filter hybridization and washing were performed as described by Patocchi et al. (1999). Signal detection was achieved by exposing Kodak $\mathrm{X}$-Omat AR autoradiographic films (Kodak, USA) to the membrane for 2 days at $-80{ }^{\circ} \mathrm{C}$.

\section{BAC DNA extraction}

A starter culture of $10 \mathrm{~mL}$ pf LB medium $(12.5 \mu \mathrm{g} / \mathrm{mL}$ chloramphenicol) was inoculated with $\mathrm{BAC}$ clones and grown for $8 \mathrm{~h}$ at $37^{\circ} \mathrm{C}$ at $340 \mathrm{rpm}$. The starter culture was used to inoculate $200 \mathrm{~mL}$ of $\mathrm{LB}$ medium $(12.5 \mu \mathrm{g} / \mathrm{mL}$ chloramphenicol), which was shaken at $340 \mathrm{rpm}$ overnight at $37{ }^{\circ} \mathrm{C}$. BAC plasmid DNA was extracted using a QIAGEN Large-Construct Kit (Qiagen, Germany) following the manufacturer's instructions and resuspended in $300 \mu \mathrm{L}$ of $\mathrm{ddH}_{2} \mathrm{O}$. DNA concentration was estimated by UV spectrometry at $260-280 \mathrm{~nm}$.

\section{BAC contig assembly}

Approximately $200 \mathrm{ng}$ of BAC plasmid DNA was digested with EcoRI and subsequently run on a $0.8 \%$ agarose gel at $100 \mathrm{~V}$ for $16 \mathrm{~h}$. Gels were stained with ethidum bromide, photographed, and blotted on Hybond-N+ membranes (GE Healthcare) as described by Patocchi et al. (1999). BAC clones showing similar digestion fingerprints and hybridization patterns were initially assigned to the same contig. Clone overlap and contig assembly were confirmed by isolating BAC-end sequences from each clone and developing 
a PCR-based assay to verify the presence or absence of the sequence in BAC clone contigs. BAC-ends were directly sequenced using BigDye Terminator Kit 3.1 (Applied Biosystems, USA) with the universal primers Sp6 and T7 close to the cloning site using $500 \mathrm{ng}$ of BAC plasmid DNA as the template. Sequencing reaction conditions consisted of 99 cycles, each cycle consisting of $30 \mathrm{~s}$ at $96{ }^{\circ} \mathrm{C}, 20 \mathrm{~s}$ at $50{ }^{\circ} \mathrm{C}$, and $4 \mathrm{~min}$ at $60{ }^{\circ} \mathrm{C}$ in a Perkin Elmer Cetus Gene Amp PCR System 9600 (Perkin Elmer). The reaction products were separated on an ABI 3100 DNA sequencing system (Applied Biosystems). Sequences were analyzed using the software Sequencer (Genecodes, USA). BAC-end sequences were initially screened for simple sequence repeats (SSRs). Specific primers were designed using Primer3 software (Rozen and Skaletsky 2000). PCRs were carried out in a final volume of $15 \mu \mathrm{L}$, as previously described for the production of the HcrVf2 probe.

\section{SSR marker development from BAC clones and mapping}

If no SSR was identified in the BAC-end sequences, SSR markers were developed from BAC clones as described by Vinatzer et al. (2004). The newly developed SSR markers (Table 1) and CH-Vf2 (Vinatzer et al. 2004) were used to genotype 254 progeny plants of a 'Fiesta' $\times$ 'Discovery' cross (Liebhard et al. 2003; Silfverberg-Dilworth et al. 2006). The CH-Vf2 SSR marker, developed from AM19-5 by Vinatzer et al. (2004), was used to orient the M18-AM19 contig (Patocchi et al. 1999). PCR conditions were as described by Silfverberg-Dilworth et al. (2006). The location of the SSRs on the genetic map was determined using JoinMap version 3.0 (Van Ooijen and Voorrips 2001). To validate whether the order of very closely linked molecular markers was supported by the presence of plants showing recombination events, the data set used for the calculation was manually analyzed in further detail. When no recombinants were found between two adjacent markers, the same map position was assigned to those markers. Although RAPD and AFLP markers were used to build the complete linkage map, these are not shown to increase the legibility of the genetic maps. The genetic maps were drawn using MapChart (Voorrips 2002).

\section{Shotgun library construction}

Approximately 5-10 $\mu \mathrm{g}$ of BAC DNA was resuspended in water in a total volume of $150 \mu \mathrm{L}$ and sheared using a Shear DNA Shearing Device (Genemachines, USA) for 30 cycles with a speed code of 10 . Blunt-end fragments were obtained by adding 10 units of Mung Bean Nuclease (New England Biolabs, USA) and $17 \mu \mathrm{L}$ of $10 \times \mathrm{MB}$ buffer to the sheared DNA sample and incubating for $30 \mathrm{~min}$ at $30{ }^{\circ} \mathrm{C}$. Sheared DNA fragments were separated on a $1 \%$ agarose gel. DNA fragments ranging from 1.5 to $3 \mathrm{~kb}$ were excised from the gel and purified using a WIZARD SV Clean-Up System Kit (Promega). Purified DNA was eluted in $50 \mu \mathrm{L}$ of $\mathrm{ddH}_{2} \mathrm{O}$ and $3 \mu \mathrm{L}$ was taken for quantification on $1 \%$ agarose gel. Dephosphorylation of the DNA sample was performed using 1 unit of calf intestinal phosphatase (New England Biolabs), $5 \mu \mathrm{L}$ of NEB buffer 3, and $7 \mu \mathrm{L}$ of $\mathrm{ddH}_{2} \mathrm{O}$ to $3 \mu \mathrm{g}$ of purified DNA in a total volume of $50 \mu \mathrm{L}$. The dephosphorylation mix was incubated for $30 \mathrm{~min}$ at $37{ }^{\circ} \mathrm{C}$. DNA was finally ethanol precipitated and

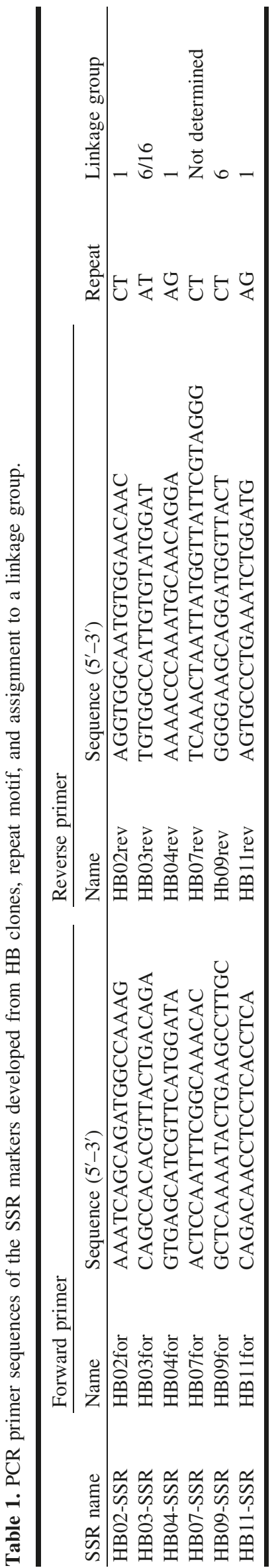

Published by NRC Research Press 
resuspended in $20 \mu \mathrm{L}$ of $\mathrm{ddH}_{2} \mathrm{O}$. BAC subfragments were cloned using a ZeroBlunt TOPO PCR Cloning Kit (Invitrogen) according to the manufacturer's instructions with slight modifications to the ligation reaction: $5 \mathrm{ng}$ of TOPO vector was used and the ligation mix was incubated overnight at room temperature. A $1 \mu \mathrm{L}$ aliquot of the ligation was mixed with $50 \mu \mathrm{L}$ of TOP10 electrocompetent Escherichia coli cells (Invitrogen, USA) and electroporation was performed using $1750 \mathrm{~V}, 25 \mu \mathrm{F}$, and $200 \Omega$.

\section{Identification of BAC subclones containing HcrVf2-like genes and sequencing}

A total of 576 single colonies $(6 \times 96$-well plates $)$ obtained from each subcloned BAC were randomly picked and resuspended in $50 \mu \mathrm{L}$ of $\mathrm{ddH}_{2} \mathrm{O}$. Cell suspensions were incubated for $10 \mathrm{~min}$ at $95{ }^{\circ} \mathrm{C}$ in a Perkin Elmer Cetus Gene Amp PCR System 9600 (Perkin Elmer) for denaturation and cell disruption. A $5 \mu \mathrm{L}$ aliquot of the solution was used as a template in PCRs containing $0.1 \mathrm{mmol} / \mathrm{L} \mathrm{dNTP}, 1 \times \mathrm{PCR}$ buffer $\left(50 \mathrm{mmol} / \mathrm{L} \mathrm{KCl}, 1.5 \mathrm{mmol} / \mathrm{L} \mathrm{MgCl}_{2}\right.$, and $10 \mathrm{mmol} /$ $\mathrm{L}$ Tris- $\mathrm{HCl}$ ) (New England Biolabs), $0.2 \mathrm{mmol} / \mathrm{L}$ each of primer (-43)M13 forward (5'-AGGGTTTTCCCAGTCACGACGTT-3') and primer (-49)M13 reverse (5'-GAGCGGATAACAATTTCACACAGG-3'), and 1.05 units of Taq DNA polymerase (New England Biolabs) in a total volume of $15 \mu \mathrm{L}$. PCR amplification was achieved performing 35 cycles, with each cycle consisting of $30 \mathrm{~s}$ at $94{ }^{\circ} \mathrm{C}, 30 \mathrm{~s}$ at $60{ }^{\circ} \mathrm{C}$, and $2.5 \mathrm{~min}$ at $72{ }^{\circ} \mathrm{C}$ followed by a final elongation step of $10 \mathrm{~min}$ at $72{ }^{\circ} \mathrm{C}$ using a Perkin Elmer Cetus Gene Amp PCR System 9600 (Perkin Elmer). A $5 \mu \mathrm{L}$ aliquot of the PCR products was separated on a $1 \%$ agarose gel to estimate insert size, while the remaining $10 \mu \mathrm{L}$ was blotted onto Hybond-N+ membranes (GE Healthcare) using a BIODOT Microfiltration Apparatus (Bio-Rad, USA) following the manufacturer's instructions. HcrVf2-like sequences were identified using the HcrVf2 sequence as a hybridization probe in Southern blot analysis following the protocol described by Patocchi et al. (1999). HcrVf2-positive subclones were grown overnight in $10 \mathrm{~mL}$ of LB medium $(50 \mu \mathrm{g} / \mathrm{mL}$ kanamycin) at $37{ }^{\circ} \mathrm{C}$. A $2 \mathrm{~mL}$ aliquot of bacterial cells was used for plasmid extraction using a GFX Micro Plasmid Prep Kit (GE Healthcare) following the manufacturer's instructions. Plasmid inserts were sequenced using (-49)M13 reverse and (-43)M13 forward primers and the BigDye Terminator Kit 3.1 (Applied Biosystems). Sequencing reactions were performed as described above for BAC-end sequences. Vector sequences were trimmed and assembly was achieved at high stringency (99\% match, 20 bp minimum overlap).

\section{Sequence analysis}

All of the generated sequences were aligned with $\mathrm{HcrVf} 2$ (GenBank accession AJ297740) using the NeedlemanWunsch global alignment present in the EMBOSS package (Rice et al. 2000). Sequences aligned to HcrVf2 but shorter than 50 nucleotides were not further considered for analysis. The other sequences were named after the BAC clone that they were isolated from, with the addition of an "s" suffix. If multiple sequences were isolated from a BAC clone, a letter was added to the name of the BAC clone. ORFs showing a length corresponding to at least one third of HcrVf2 and aligning in the sense orientation of the HcrVf2 ORF were identified with CLC DNA workbench software (available from www.clcbio.com) and aligned to the HcrVf2 ORF using Dialign (Morgenstern 2004). The quality of the alignment was evaluated with SWAAP (Pride 2000), calculating identities at the nucleotide and amino acid level. A maximum likelihood phylogenetic tree was constructed from the aligned sequences using the Jukes and Cantor method (Jukes and Cantor 1969) and RDP2 (Martin et al. 2005). The resulting radiation tree was drawn using MEGA 4.0 software (Tamura et al. 2007).

\section{Expression analysis}

The expression of HcrVf2 paralogs with the complete ORF structure was assessed by RT-PCR in young uninoculated apple leaves of 'Florina'. To identify each RGA gene unequivocally, ORF sequences were aligned and specific PCR primer pairs were designed for each paralog (see Table 4). Total RNA was extracted from leaves using a SV Total RNA Extraction Kit (Promega) following the manufacturer's instructions. Single-strand cDNA was synthesized using a RevertAid ${ }^{\mathrm{TM}}$ First Strand cDNA Synthesis Kit (Fermentas, USA) following the manufacturer's protocol and using Oligo $\mathrm{d}(\mathrm{T})_{18}$ as the primer for the synthesis. Single-strand cDNA was used as a template for PCR amplification using the specific primers designed for each RGA gene. The PCR consisted of $1 \mu \mathrm{L}$ of cDNA, $1 \times$ Taq buffer, $0.2 \mu \mathrm{mol} / \mathrm{L}$ of each primer, $0.1 \mathrm{mmol} / \mathrm{L} \mathrm{dNTPs}$, and $0.7 \mathrm{unit}$ of Taq DNA polymerase (New England Biolabs) in a total volume of $10 \mu \mathrm{L}$. Amplification was carried out for 35 cycles of $94{ }^{\circ} \mathrm{C}$ for $30 \mathrm{~s}, 60{ }^{\circ} \mathrm{C}$ for $30 \mathrm{~s}$, and $72{ }^{\circ} \mathrm{C}$ for 2 min. PCR products were separated by electrophoresis on a $1 \%$ agarose gel and visualized by ethidium bromide staining.

\section{Results}

\section{Identification of $\mathrm{BAC}$ clones carrying $\mathrm{Hcr} V \boldsymbol{f} 2$-like sequences}

A total of 30 clones, out of 36864 of the entire BAC library, were found to hybridize to the HcrVf2 probe. The first 19 BAC clones (clones AL07-2, AL07-3, AL07S-3, AM19-1, AM19-2, AM19-3, AM19-4, AM19-5, M18-1, M18-2, M18-3, M18-4, M18-5, M18-6, M18-7, M18S-1, M18S-3, M18S-4, and M18S-5) were already assembled in contigs and named after Patocchi et al. (1999). The other 11 BAC clones, with an unknown assembly and map position, were named HB01 (homologous BAC 01) through HB11 for simplicity (BAC clones 15J14, 43F22, 40O10, 49J15, 55I7, 57G7, 62H3, 72M18, 76G16, 90N4, and 94H24).

\section{Assembly, mapping, and selection of HB BAC clones for subcloning}

The EcoRI restriction pattern of the $11 \mathrm{HB}$ BAC clones was used for fingerprinting and, together with PCR markers developed from each BAC-end sequence, was used to assemble HB clones into contigs. The HB10 clone was not viable; therefore, it was no longer analyzed.

Three contigs were assembled. The first contig consisted of clones HB01, HB05, and HB11, and an SSR marker, HB11-SSR, developed from HB11 (Table 1), mapped this contig $21 \mathrm{cM}$ from the $V f$ locus (locus CH-Vf1, Fig. 1). Clones HB01 and HB11 represented the entire contig and 
Fig. 1. Contigs and map position of the BAC clones containing HcrVf analogs. LG1, LG6, and LG16 are the genetic maps (SSR backbones) of linkages 1, 6, and 16 of 'Discovery'. In bold are represented newly developed SSR markers (CH-Vf1 and CH-Vf2 from Vinatzer et al. 2004). Genetic distances are cumulative and expressed as cM. A questionmark indicates that the position of the HB04 BAC clone could not be determined with respect to the AL07 contig. Shaded bars, BAC clones hybridizing to HcrVf2; open bars, BAC clones of the M18-AM19 and AL07 contigs developed by Patocchi et al. (1999) not hybridizing to HcrVf2. A solid box is used to mark the position on the contigs of sequences showing all of the domains predicted in HcrVf2 and expressed in young leaves of 'Florina' (not inoculated with apple scab). The broken boxes indicate the positions on the contigs of sequences showing all of the domains found in HcrVf2 but not expressed in young leaves of 'Florina'.

LG1

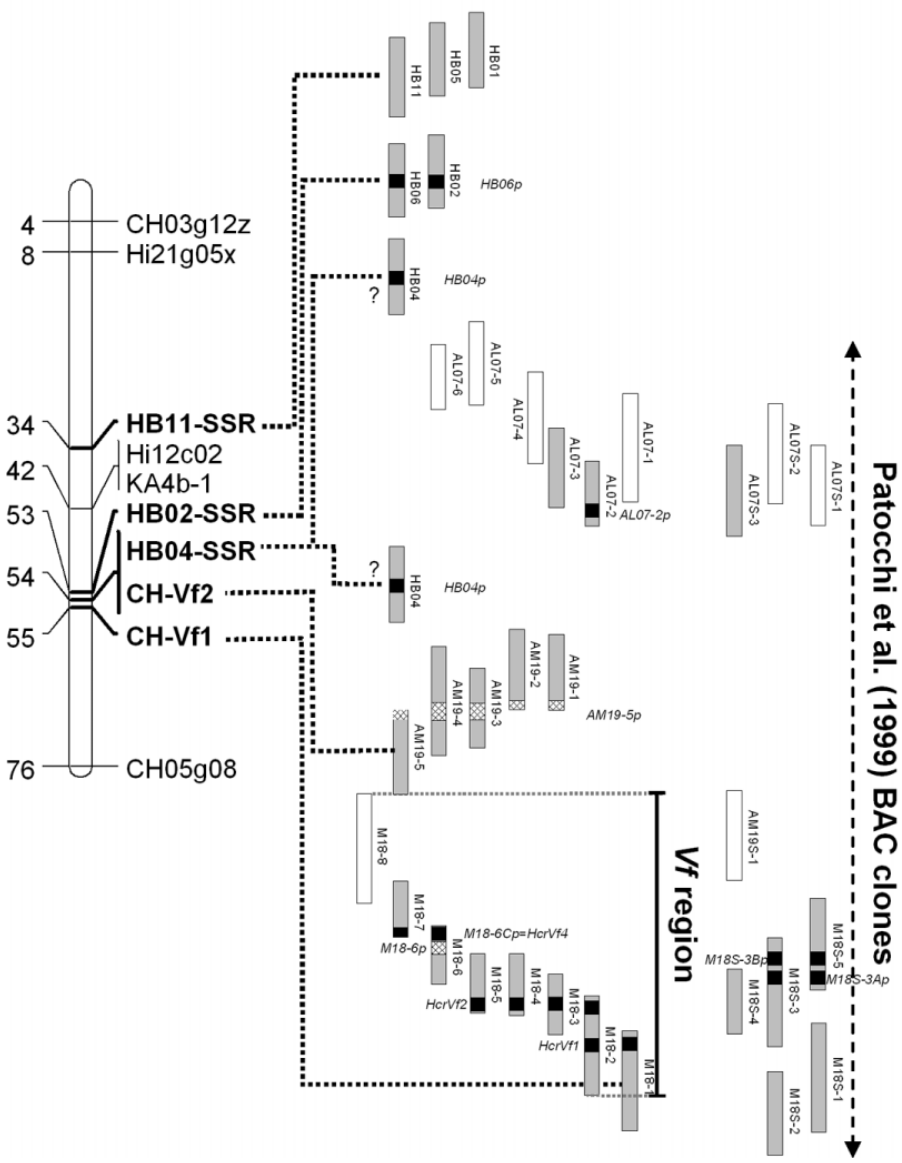

were chosen for shotgun cloning. The second contig consisted of clones HB02 and HB06, and the SSR marker identified on HB02 (Table 1) positioned this contig $2 \mathrm{cM}$ from the $V f$ locus. These two clones were found to share the same EcoRI fragment hybridizing to the HcrVf2 probe (data not shown). Therefore, only HB06 was sufficient to identify all of the HcrVf paralogs of this contig. Finally, HB03 and HB08 constituted the third contig, which was mapped due to a multilocus SSR marker on HB03 (Table 1). HB03SSRy is located at the top of LG6, while HB03-SSRx maps at the bottom of LG16. HB03-SSR was also mapped using a small subset of a 'Florina' $\times$ 'Nova Easygro' progeny. Since 'Florina' is the cultivar used for the construction of the BAC library, it was possible to ascertain that the HB03-HB08 contig originates from LG6. The BAC clones HB03 and HB08 also shared the same EcoRI fragment hybridizing to the HcrVf2 probe (data not shown), and therefore, only HB03 was selected to identify all of the HcrVf paralogs of this contig.

The remaining BAC clones, HB04, HB07, and HB09,
LG6

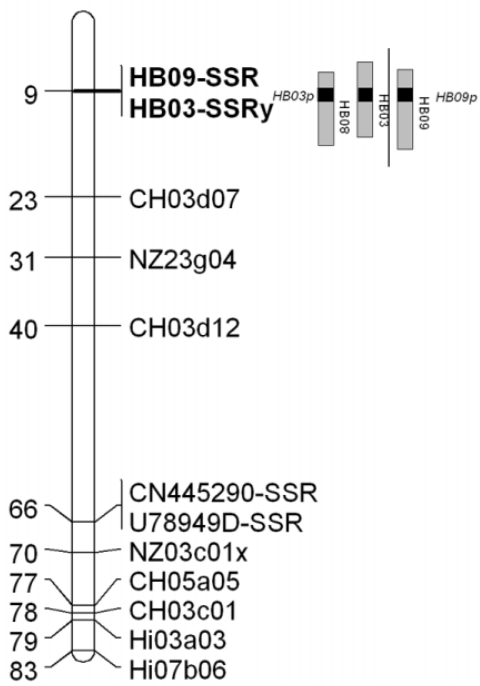

LG16
LG6 Contigs

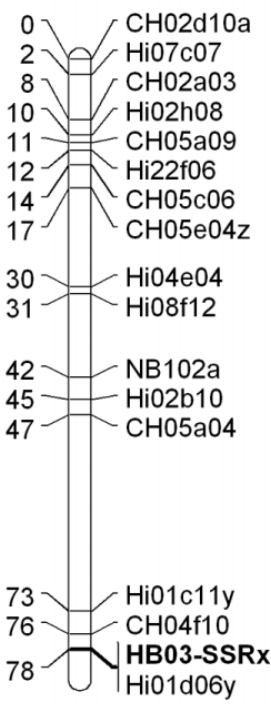

- complete ORFs expressed in young leaves

* complete ORFs not expressed in young leaves

$\checkmark$ sequencing incomplete

were found to be singletons. SSR markers HB04-SSR, HB07-SSR, and HB09-SSR were developed from BAC clones HB04, HB07, and HB09, respectively (Table 1). HB04-SSR was mapped at $1 \mathrm{cM}$ from the $V f$ locus; BAC clone HB07 could not be mapped, as the SSR marker derived from this clone was not polymorphic in the cross 'Fiesta' $\times$ 'Discovery' or in 'Florina' $\times$ 'Nova Easygro'. HB09-SSR was found to cosegregate with the HB03-SSR locus $y$ at the top of LG6.

The CH-Vf2 marker, developed by Vinatzer et al. (2004) from clone AM19-5 of the M18-AM19 contig, cosegregated with HB04-SSR at $1 \mathrm{cM}$ from CH-Vf1, allowing the orientation of the M18-AM19 contig to be defined. As the AL07 contig was not mapped, it was not possible to establish the exact location of the $\mathrm{HB} 04$ BAC clone in relation to the AL07 contig.

\section{Identification of $\mathrm{HcrVf}$ paralogs}

A large number of BAC clones from the $V f$ region were positive for the HcrVf2 probe using hybridization analysis. 
Excluding the BAC clone belonging to the $V f$ region of the resistant chromosome of 'Florina' studied by Vinatzer et al. (2001), the BAC clones HB01, HB03, HB04, HB06, HB09, HB11, AL07-2, AL07-3, AM19-5, AM19-1, M18-6, M18S3 , and M18S-5 were investigated for the presence of HcrVf2 paralogs.

The 13 selected BAC clones were subcloned and 22 sequences showing similarity to $H c r V f 2$ were identified. Sequence analysis could not identify an ORF similar to HcrVf2 for nine sequences. The M18S-5Bs sequence could not be completed either by direct sequencing or by subclone sequencing, and since no ORF could be readily identified, it was excluded from further analysis, even if showing a high level of similarity to the HcrVf2 gene. The remaining 12 proteins were designated after the BAC clone from which they originated, with the addition of a " $p$ " suffix for "paralog". The predicted protein structure of the HcrVf2 paralogs identified in the 12 sequences showed that nine $(H B O 3 p$, HB04p, HB06p, HB09p, M18-6Cp, M18S-3Ap, M18S-3Bp, $A L 07-2 p$, and $A M 19-5 p$ ) encode proteins containing all of the seven domains predicted for the HcrVf2 gene (domains A-G), while three proteins (M18S-5p, M18S-3Cp, and M18$6 p$ ) represent truncated versions of the gene (Table 2). In the sequence M18S-5s encoding the M18S-5p protein, a $2 \mathrm{bp}$ deletion caused a frame-shift mutation leading to the formation of a premature stop codon at the end of the $\mathrm{C}$ domain (Fig. 2). This was the only frame-shift mutation observed in the gene family. M18-6p and M18S-3Cp both lack the domains $\mathrm{A}$ and $\mathrm{B}$, but $M 18-6 p$ is additionally prematurely terminated by a 778 bp insertion that introduced a stop codon towards the end of the domain C. Therefore, M18-6p lacks the domains A, B, and D-G (Fig. 2).

\section{Gene family diversity}

Nucleotide sequence identity between ORF encoding RGAs and HcrVf2 varied between $73 \%$ and $94 \%$, with an average of $89 \%$. At the amino acid level, the identity ranged from $63 \%$ to $89 \%$, with an average of $84 \%$ (Table 3). The genes showing the highest identity with HcrVf2 were M18$6 C p$ (identical to HcrVf4, see Discussion), HcrVfl, and $M 18 S-3 B p$ with up to $94 \%$ identity at the nucleotide level and $89 \%$ at the amino acid level. The ORF with the lowest identity was $H B 06 p$ with $73 \%$ and $63 \%$ at the nucleotide and amino acids levels, respectively. The most evident differences observed among the paralogs concerned the number of LRRs, which ranged from 26 to 31 (Fig. 2).

A dendrogram was generated from the aligned nucleotide sequences of $11 \mathrm{HcrVfs}$, including the nine identified in this study, HcrVf1, and HcrVf2 (Fig. 3). Three clades were distinguished. The first clade, designated " $V f$ clade", consisted of ORFs from the $V f$ region (HcrVf1, HcrVf2, HcrVf4, MI8S$3 A p$, and $M 18 S-3 B p)$, regardless of whether they were present on the "resistant" (carrying the $V f$ gene) or "susceptible" homologous chromosomes. The second clade was composed of the AM19-5p and AL07-2p genes, which were found on the AL07 and AM19 contigs, respectively. The last clade grouped together the HBO4p, HBO3p, and HBO9p sequences, deriving from LG1 and LG6.

\section{Expression analysis of $\mathrm{HcrVf}$ paralogs}

Specific PCR primers were designed for paralogs identi- fied as having a complete ORF (Table 4). Transcription in young uninfected apple leaves of 'Florina' was verified by RT-PCR for the paralogs HB03p, HB04p, M18S-3Ap, $M 18 S-3 B p, A L 07-2 p$, and HB06p. No transcripts were detected for the HB09p and AM19-5p genes (Fig. 4).

\section{Discussion}

The HcrVf2 gene is currently the only HcrVf gene whose function as an apple scab $\mathrm{R}$ gene has been unambiguously demonstrated by the transformation of a susceptible cultivar (Belfanti et al. 2004; Malnoy et al. 2008). We hypothesized that the identification and mapping of HcrVf2 paralogous sequences may provide a way to accelerate the cloning of additional $\mathrm{R}$ genes and the development of molecular markers tightly associated with them. Therefore, we investigated the distribution of HcrVf2 paralogs in the apple genome by screening a BAC library of the apple cultivar 'Florina' using the whole $H c r V f 2$ gene sequence as a hybridization probe, retrieving 30 BAC clones. Three additional BAC clones (AM19-1, AM19-2, and M18-7) were detected compared with the 27 BAC clones presented by Vinatzer et al. (2001). In that study, the sequence of AL07-2-Sp6, which is 93\% identical to the last two LRRs and to the D and E domains of the HcrVf2 gene, was used as a hybridization probe. These three BAC clones were not detected, as they carried only partial HcrVf sequences, which are lacking the part matching to the probe AL07-2-Sp6.

Vinatzer et al. (2001) published the sequence of HcrVfl, $H c r V f 2$, and HcrVf3 and reported that a fourth HcrVf (HcrVf4) was present on the BAC clone M18-6. However, the sequence of HcrVf3 was not confirmed by sequencing genomic clones (as was the case for HcrVfl and HcrVf2) and only a partial genomic sequence of HcrVf4 was identified. In this study, we were able to identify the complete genomic sequence of HcrVf4 (corresponding to M18-6Cp) but unable to isolate HcrVf3. Since the HcrVf3 sequence (AJ297741) described by Vinatzer et al. (2001) was PCR derived, we hypothesize that $H c r V f 3$ might in fact be a PCR artifact. Xu and Korban (2002), in their effort to identify $V f$ candidate genes, sequenced BAC clones derived from the $V f$ region. They described four genes, which they named Vfal$V f a 4$. Vfal and Vfa2 were identical to HcrVfl and HcrVf2, while Vfa3 had multiple truncated transcripts and an insertion of $780 \mathrm{bp}$. Interestingly, M18-6p contains a $778 \mathrm{bp}$ insertion, similar to the one described for $V f a 3$, and therefore, $M 18-6 p$ probably represents $V f a 3$. Vfa4 is reported to be 2748 bp long, while HcrVf4 is 2889 bp long. This difference of $141 \mathrm{bp}$ can be explained by the fact that the first start codon that we identified for HcrVf4 is 141 bp upstream from the "normal" start codon of HcrVf2 and all of the other $H c r V f 2$ paralogs. It is likely that this first start codon that we identified still belongs to the transcription regulatory sequence and will not initiate the translation of the gene. In fact, Xu and Korban (2002) did not identify this sequence from their RACE experiment. Unfortunately, Vfa3 and Vfa4 sequences are not publicly available; therefore, it is not possible to verify whether those sequences are in fact the same genes as M18-6p and HcrVf4, respectively.

The clades of the dendrogram generated from the alignment of the $11 \mathrm{HcrVf}$ sequences reflect their organization 
Table 2. HcrVf2 paralogs showing sequence length, sequence accession number, presence of an ORF with (+) or without (-) all seven domains of the HcrVf2 gene, names of the HcrVf2 paralogs, and the presence (+) or absence (-) of transcripts in young uninfected 'Florina' leaves.

\begin{tabular}{|c|c|c|c|c|c|c|}
\hline $\begin{array}{l}\text { Sequence } \\
\text { name }\end{array}$ & $\begin{array}{l}\text { Sequence } \\
\text { length } \\
\text { (bp) }\end{array}$ & $\begin{array}{l}\text { Sequence ac- } \\
\text { cession No. }\end{array}$ & $\begin{array}{l}\text { ORF length } \\
\text { (bp) }\end{array}$ & $\begin{array}{l}\text { Presence-absence } \\
\text { of all HcrVf2 do- } \\
\text { mains }\end{array}$ & $\begin{array}{l}\text { HcrVf2 paralog } \\
\text { name }\end{array}$ & Transcription \\
\hline M18S-5As & 3896 & EU794454 & 2370 & - & $M 18 S-5 p$ & \\
\hline M18S-3As & 3938 & EU794450 & 3135 & + & $M 18 S-3 A p$ & + \\
\hline M18S-3Bs & 3840 & EU794457 & 2901 & + & $M 18 S-3 B p$ & + \\
\hline M18S-3Cs & 4002 & EU794458 & 2409 & - & $M 18 S-3 C p$ & \\
\hline M18-6As & 5792 & EU794446 & 1836 & - & M18-6p & \\
\hline M18-6Cs & 4299 & EU794466 & 2889 & + & $M 18-6 C p^{a}$ & $+^{b}$ \\
\hline HB09s & 3118 & EU794461 & 2925 & + & $H B 09 p$ & - \\
\hline HB06-As & 4416 & EU794459 & 2895 & + & HB06 $p$ & + \\
\hline $\mathrm{HB} 04 \mathrm{~s}$ & 6824 & EU794453 & 2931 & + & $H B 04 p$ & + \\
\hline HB03s & 4410 & EU794449 & 2922 & + & $H B 03 p$ & + \\
\hline AM19-5s & 8134 & EU794448 & 3114 & + & $A M 19-5 p$ & - \\
\hline AL07-2As & 5911 & EU794451 & 3123 & + & $A L 07-2 p$ & + \\
\hline
\end{tabular}

Note: Nucleotide and amino acid sequences have been submitted to the NCBI database as accession Nos. EU794445 to EU794466.

${ }^{a}$ Sequence corresponding to HcrVf4.

${ }^{b}$ Data from Vinatzer et al. (2001).

Fig. 2. Schematic representations of the $\mathrm{HcrVf}$ proteins. Protein domains: A, predicted signal peptide (not shown); $\mathrm{B}, \mathrm{NH}_{2}$ terminus of the mature protein; C, extracellular leucine-rich repeat (LRR) domain; D, domain with an unknown function; E, acidic domain; F, hydrophobic transmembrane domain; $\mathrm{G}$, basic $\mathrm{C}$ terminus of the protein. The number of LRR motifs is indicated below each putative protein structure.

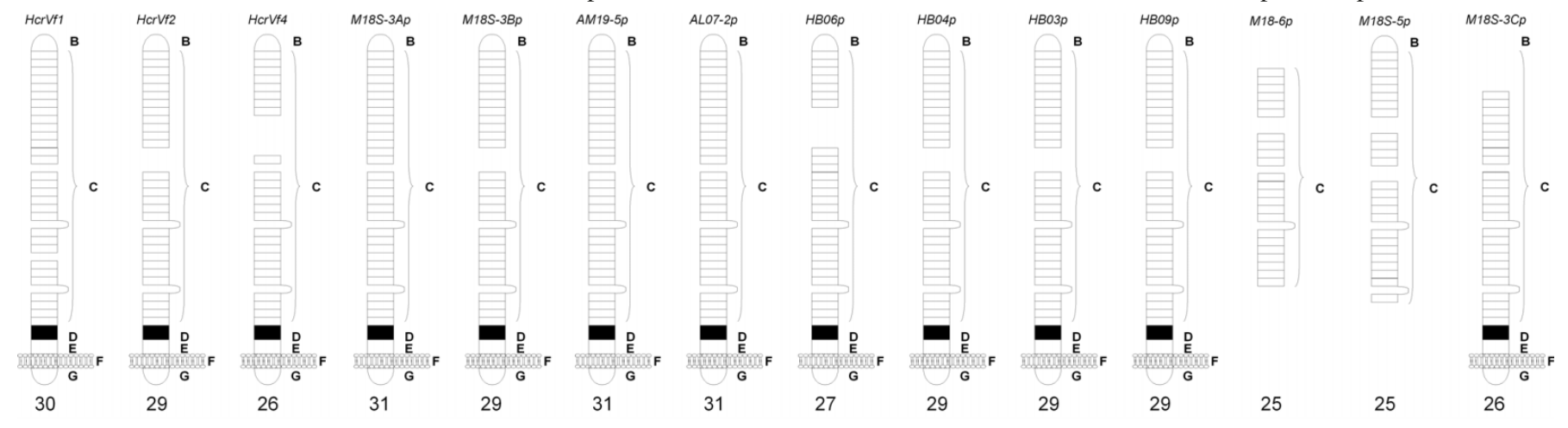

Table 3. Similarity of the HcrVf2 gene to the other $H c r V f$-like genes at the nucleotide and amino acid level calculated with SWAAP.

\begin{tabular}{lll}
\hline & $\begin{array}{l}\% \text { nucleotide } \\
\text { identity }\end{array}$ & $\begin{array}{l}\% \text { amino } \\
\text { acid identity }\end{array}$ \\
\hline Hcrvf2 vs. $H c r V f 1$ & 93.2 & 88.7 \\
Hcrvf2 vs. $M 18-6 C p^{a}$ & 93.7 & 89.0 \\
Hcrvf2 vs. $M 18 S-3 A p$ & 91.9 & 86.5 \\
Hcrvf2 vs. $M 18 S-3 B p$ & 93.5 & 88.6 \\
Hcrvf2 vs. $H B 09 p$ & 90.4 & 84.4 \\
Hcrvf2 vs. $H B 06 p$ & 73.3 & 63.6 \\
Hcrvf2 vs. $H B 04 p$ & 91.8 & 86.6 \\
Hcrvf2 vs. $H B 03 p$ & 90.5 & 83.9 \\
Hcrvf2 vs. $A M 19-5 p$ & 88.6 & 82.0 \\
Hcrvf2 vs. $A L 07-2 p$ & 90.6 & 84.7 \\
Average & $89.8 \pm 6$ & $83.8 \pm 7.5$ \\
\hline
\end{tabular}

${ }^{a}$ Identical to HcrVf4. within the apple genome. The sequences of the genes derived from the $V f$ region, regardless of whether they derived from BAC clones of the "resistant" chromosome (HcrVfl, $H c r V f 2$, and HcrVf4) or from the corresponding region of the "susceptible" homologous chromosome (M18S-3Ap and $M 18 S-3 B p$ ), were grouped into a single clade ("Vf clade"). The phylogenetic distance and physical position in the apple genome of $M 18 S-3 A p$ and $M 18 S-3 B p$ suggest that these paralogs might be alleles of HcrVf1, HcrVf2, or HcrVf4. Diversity between HcrVf paralogs increased with the genetic distance along the chromosome. In fact, the paralogs with the smallest phylogenetic distance from the $V f$ clade (ALO7$2 p$ and $A M 19-5 p$ ) map within $1 \mathrm{cM}$ of the $V f$ locus, as does $H B 04 p$, belonging to the second closest clade. The most diverse gene, $H B 06 p$, mapped at the farthest genetic distance from the $V f$ locus (about $2 \mathrm{cM}$ ). Interestingly, on the HB01-HB05-HB11 contig located about $21 \mathrm{cM}$ away from $V f$, only short sequences having similarity to the HcrVf2 gene were identified. Therefore, it is not inconceivable that early separation of the two gene clusters allowed an evolutionary differentiation process to proceed, leading to the ac- 
Fig. 3. Maximum likelihood tree generated from the alignment of the HcrVf-like sequences encoding for proteins showing all of the domains described for HcrVf2.

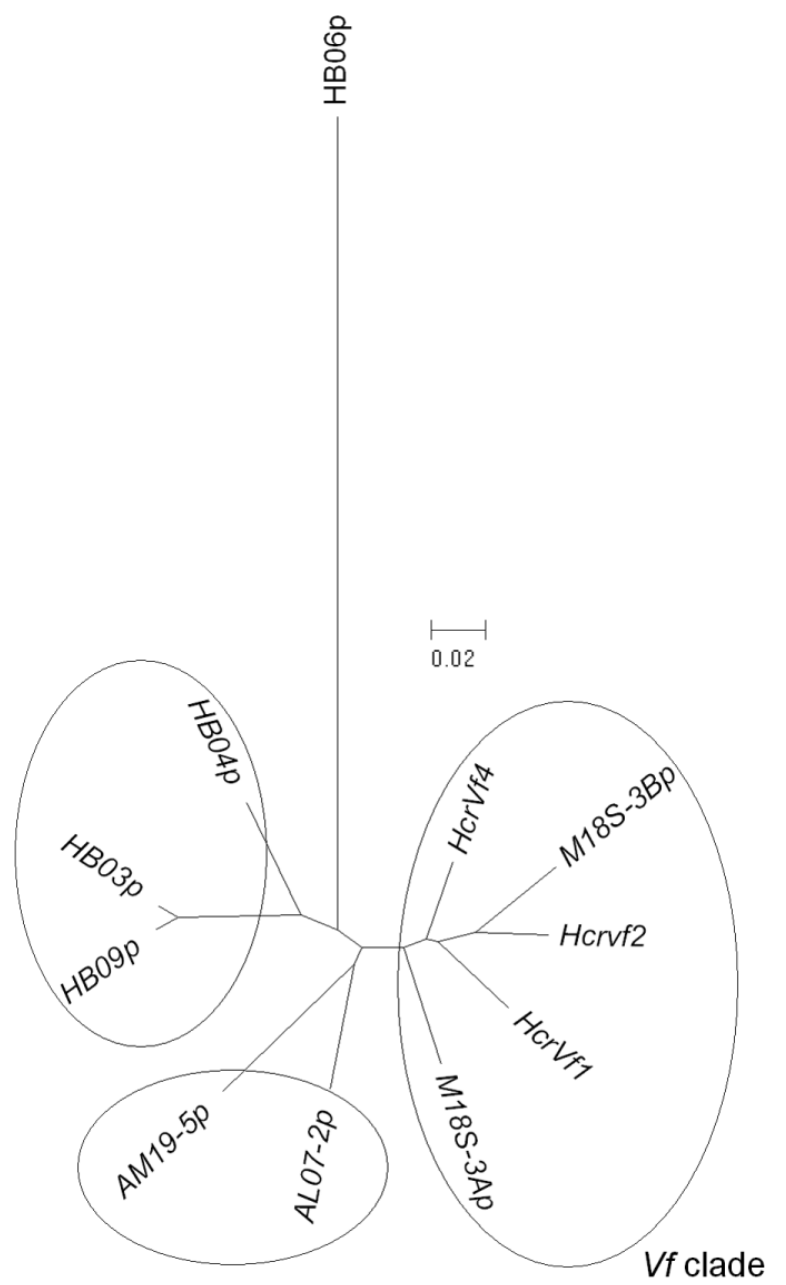

cumulation of a large number of mutations in the locus at $21 \mathrm{cM}$ from $V f$, which was released from selective pressure by sequence duplication.

The paralogs located at the top of LG6 can provide further insight into the evolution of the gene family. HB03 and HB09 BAC clones do not belong to the same contig because their restriction profile and hybridization pattern are slightly different; however, they share very similar BAC-end sequences (data not shown) and map at the same location on LG6. Therefore, it is reasonable to assume that HB03 and HB09 represent allelic variants. Moreover, considering the similarity of $\mathrm{HBO3p}$ and $\mathrm{HBO} 0 \mathrm{p}$ with $\mathrm{HBO4p}$, it is possible that they derived from a single duplication event of the $H B 04 p$ gene. It is likely that such an event would have been rather recent, since the HBO4p and HBO9p genes share high sequence similarity.

The distribution of the HcrVf genes in the apple genome is similar to that described for the $C f \mathrm{R}$ genes of tomato (also LRR-TM type of R genes). In both species, LRR-TM genes were found on only two linkage groups. In tomato, three clusters of the Hcr9 (homologous to the Cf9 gene) and Cf4/9 genes, named Southern Cross, Milky Way, and Northern Lights, were mapped on tomato LG1 (Parniske et

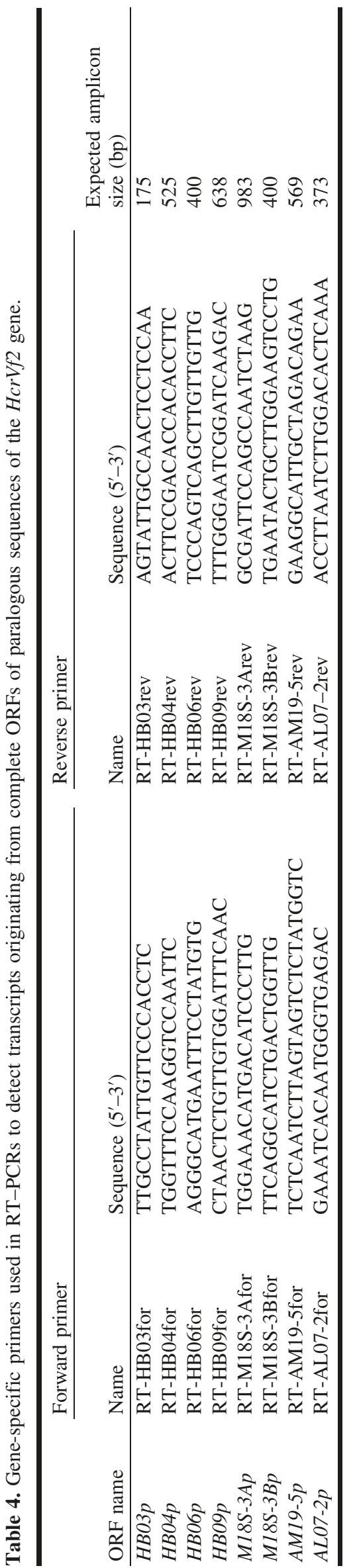

Published by NRC Research Press 
Fig. 4. Transcription analysis of HcrVf genes by RT-PCR in young uninfected leaves of 'Florina'. RT, specific PCR amplification using cDNA as a template; +, amplification from BAC clone DNA; -, negative control obtained by PCR amplification with HB04p specific primers using the extracted RNA as a template to confirm the absence of genomic DNA contamination.

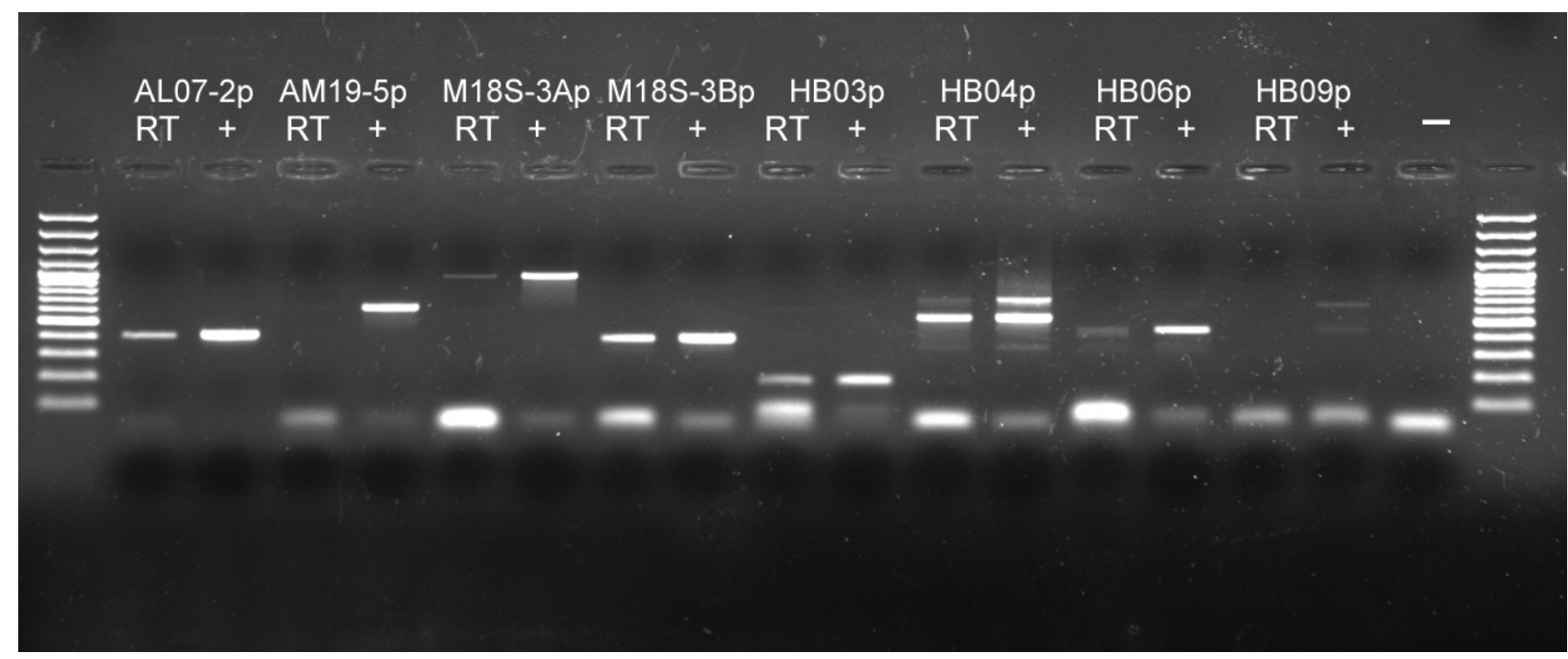

al. 1999). A second cluster, containing $C f 2$ and $C f 5$, was identified on LG6. Similarly, in apple, three loci containing full-length HcrVf paralogs and the $V f$ locus were identified on LG1, while a single cluster was present on chromosome 6. However, in contrast with what has been described in tomato, a single complete HcrVf gene was identified at each locus, instead of a gene cluster, with the only exception of the Vf region, where HcrVfl, HcrVf2, and HcrVf4 were present. Dixon et al. (1996) suggested that the tomato Hcr9 and $\mathrm{Hcr} 2$ families derived from a common ancestor gene and that these families evolved independently to give rise to different gene families. A similar hypothesis can be postulated for the HcrVf genes in apple where the family may have evolved from a single progenitor located on chromosome 1 and duplicated on chromosome 6 . The absence of a gene cluster on LG6 in apple seems to corroborate the hypothesis that the duplication or translocation event occurred recently and has not had the opportunity to evolve further paralogous sequences during a relatively short evolutionary time frame. Interestingly, besides structural similarities between the $H c r V f 2$ and $C f 9$ proteins, this study provides evidence for a similar genomic organization and a comparable evolutionary descent of this class of $\mathrm{R}$ genes in tomato and apple, which are taxonomically distant species.

Several apple scab $\mathrm{R}$ genes have been identified and mapped in apple (reviewed in Gessler et al. 2006). However, none of them cosegregate with any of the HcrVf2-like genes identified in this study. The apple scab $\mathrm{R}$ gene closest to an HcrVf-like gene is Rvil4, identified in a population derived from 'Dülmener Rosenapfel' and located $4 \mathrm{cM}$ from HB09SSR on LG6 (Soufflet-Freslon et al. 2008). Therefore, the apple scab $\mathrm{R}$ genes identified and mapped to date do not have high sequence similarity with $H c r V f 2$ and supposedly either belong to different $\mathrm{R}$ gene classes or have sufficiently diverged from HcrVf2. Therefore, it is reasonable to assume that resistance against apple scab originated independently and resistance to the same pathogen can be achieved by dif- ferent mechanisms. The exploitation of gene diversity and redundancy within the family members of each class, combined with the possibility of rapidly generating variability through a cluster arrangement of the genes, might have played a decisive role in evolution and allowed the host plant to cope with large, rapidly evolving pathogen populations. From the practical point of view, the fact that resistance against apple scab could be obtained using different classes of $\mathrm{R}$ genes gives a higher chance that gene pyramiding strategies aimed at obtaining durable resistance might be effective.

\section{Acknowledgements}

The authors are grateful to Prof. Beat Keller for allowing access to the facilities of his research group and Dr. Eve Silfverberg-Dilworth for the critical reading of this manuscript. Cristina Durante and Simona Morello are gratefully acknowledged for their technical assistance. The work has been financed in part by ETH internal grant TH-11/04-2.

\section{References}

Baldi, P., Patocchi, A., Zini, E., Toller, C., Velasco, R., and Komjanc, M. 2004. Cloning and linkage mapping of resistance gene homologues in apple. Theor. Appl. Genet. 109: 231-239. doi:10. 1007/s00122-004-1624-x. PMID:15052401.

Belfanti, E., Silfverberg-Dilworth, E., Tartarini, S., Patocchi, A., Barbieri, M., Zhu, J., et al. 2004. The HcrVf2 gene from a wild apple confers scab resistance to a transgenic cultivated variety. Proc. Natl. Acad. Sci. U.S.A. 101: 886-890. doi:10.1073/pnas. 0304808101. PMID:14715897.

Braun, T., Schofield, P.R., and Sprengel, R. 1991. Amino-terminal leucine-rich repeats in gonadotropin receptors determine hormone selectivity. EMBO J. 10: 1885-1890. PMID:2050124.

Calenge, F., Van der Linden, C.G., Van de Weg, E., Schouten, H.J., Van Arkel, G., Denance, C., and Durel, C.E. 2005. Resistance gene analogues identified through the NBS-profiling method map close to major genes and QTL for disease resis- 
tance in apple. Theor. Appl. Genet. 110: 660-668. doi:10.1007/ s00122-004-1891-6. PMID:15647920.

Collins, N.C., Webb, C.A., Seah, S., Ellis, J.G., Hulbert, S.H., and Pryor, A. 1998. The isolation and mapping of disease resistance gene analogs in maize. Mol. Plant-Microbe Interact. 11: 968978. doi:10.1094/MPMI.1998.11.10.968. PMID:9768514.

Dixon, M.S., Jones, D.A., Keddie, J.S., Thomas, C.M., Harrison, K., and Jones, J.D.G. 1996. The tomato Cf-2 disease resistance locus comprises two functional genes encoding leucine-rich repeat proteins. Cell, 84: 451-459. doi:10.1016/S0092-8674(00) 81290-8. PMID:8608599.

Flor, H.H. 1971. Current status of gene-for-gene concept. Annu. Rev. Phytopathol. 9: 275-296. doi:10.1146/annurev.py.09. 090171.001423.

Gardiner, S., Murdoch, J., Meech, S., Rusholme, R., Bassett, H., Cook, M., et al. 2003. Candidate resistance genes from an EST database prove a rich source of markers for major genes conferring resistance to important apple pests and diseases. Acta Hortic. 622: 141-151.

Gessler, C., Patocchi, A., Sansavini, S., Tartarini, S., and Gianfranceschi, L. 2006. Venturia inaequalis resistance in apple. Crit. Rev. Plant Sci. 25: 473-503. doi:10.1080/07352680601015975.

Jones, D.A., Thomas, C.M., Hammondkosack, K.E., Balintkurti, P.J., and Jones, J.D.G. 1994. Isolation of the tomato Cf-9 gene for resistance to Cladosporium fulvum by transposon tagging. Science (Washington, D.C.), 266: 789-793. doi:10.1126/science. 7973631. PMID:7973631.

Joosten, M., and de Wit, P. 1999. The tomato-Cladosporium fulvum interaction: a versatile experimental system to study plantpathogen interactions. Annu. Rev. Phytopathol. 37: 335-367. doi:10.1146/annurev.phyto.37.1.335. PMID:11701827.

Jukes, T., and Cantor, C. 1969. Evolution of protein molecules. In Mammalian protein metabolism. Edited by H.N. Munro. Academic Press, New York. pp. 21-132.

Kruijt, M., De Kock, M., and De Wit, P. 2005. Receptor-like proteins involved in plant disease resistance - review. Mol. Plant Pathol. 6: 85-97. doi:10.1111/j.1364-3703.2004.00264.x.

Leister, D., Ballvora, A., Salamini, F., and Gebhardt, C. 1996. A PCR-based approach for isolating pathogen resistance genes from potato with potential for wide application in plants. Nat. Genet. 14: 421-429. doi:10.1038/ng1296-421. PMID:8944022.

Liebhard, R., Koller, B., Gianfranceschi, L., and Gessler, C. 2003. Creating a saturated reference map for the apple (Malus $\times$ domestica Borkh.) genome. Theor. Appl. Genet. 106: 14971508. PMID:12677403.

MacHardy, W.E. 1996. Apple scab biology, epidemiology and management. APS Press, St. Paul, Minn.

Malnoy, M., Xu, M., Borejsza-Wysocka, E., Korban, S.S., and Aldwinckle, H.S. 2008. Two receptor-like genes, Vfal and Vfa2, confer resistance to the fungal pathogen Venturia inaequalis inciting apple scab disease. Mol. Plant-Microbe Interact. 21: 448458. doi:10.1094/MPMI-21-4-0448. PMID:18321190.

Martin, D.P., Williamson, C., and Posada, D. 2005. RDP2: recombination detection and analysis from sequence alignments. Bioinformatics, 21: 260-262. doi:10.1093/bioinformatics/bth490. PMID:15377507.

Morgenstern, B. 2004. DIALIGN: multiple DNA and protein sequence alignment at BiBiServ. Nucleic Acids Res. 32: W33W36. doi:10.1093/nar/gkh373. PMID:15215344.

Naik, S., Hampson, C., Gasic, K., Bakkeren, G., and Korban, S.S. 2006. Development and linkage mapping of E-STS and RGA markers for functional gene homologues in apple. Genome, 49: 959-968. doi:10.1139/G06-085. PMID:17036071.

Parniske, M., Wulff, B.B.H., Bonnema, G., Thomas, C.M., Jones,
D.A., and Jones, J.D.G. 1999. Homologues of the Cf-9 disease resistance gene $(\mathrm{Hcr} 9 \mathrm{~s})$ are present at multiple loci on the short arm of tomato chromosome 1. Mol. Plant-Microbe Interact. 12: 93-102. doi:10.1094/MPMI.1999.12.2.93. PMID:9926411.

Patocchi, A., Vinatzer, B.A., Gianfranceschi, L., Tartarini, S., Zhang, H.B., Sansavini, S., and Gessler, C. 1999. Construction of a $550 \mathrm{~kb}$ BAC contig spanning the genomic region containing the apple scab resistance gene $V f$. Mol. Gen. Genet. 262: 884891. doi:10.1007/s004380051154. PMID:10628874.

Pride, D.T. 2000. SWAAP version 1.0.0 — sliding windows alignment analysis program: a tool for analyzing patterns of substitutions and similarity in multiple alignments. Distributed by the author at www.bacteriamuseum.org/SWAAP/SwaapPage.htm.

Rice, P., Longden, I., and Bleasby, A. 2000. EMBOSS: the European molecular biology open software suite. Trends Genet. 16: 276277. doi:10.1016/S0168-9525(00)02024-2. PMID:10827456.

Rozen, S., and Skaletsky, H.J. 2000. Primer3 on the WWW for general users and for biologist programmers. In Bioinformatics methods and protocols. Edited by S. Misener and S. Krawetz. Humana Press, Totowa, N.J. pp. 365-386.

Shen, K.A., Meyers, B.C., Islam-Faridi, M.N., Chin, D.B., Stelly, D.M., and Michelmore, R.W. 1998. Resistance gene candidates identified by PCR with degenerate oligonucleotide primers map to clusters of resistance genes in lettuce. Mol. Plant-Microbe Interact. 11: 815-823. doi:10.1094/MPMI.1998.11.8.815. PMID:9675895.

Silfverberg-Dilworth, E., Matasci, C.L., Van de Weg, W.E., Van Kaauwen, M.P.W., Walser, M., Kodde, L.P., et al. 2006. Microsatellite markers spanning the apple (Malus $\times$ domestica Borkh.) genome. Tree Genet. Genomes, 2: 202-224. doi:10.1007/ s11295-006-0045-1.

Soufflet-Freslon, V., Gianfranceschi, L., Patocchi, A., and Durel, C.E. 2008. Inheritance studies of apple scab resistance and identification of Rvi14, a new major gene that acts together with other broad-spectrum QTL. Genome, 51: 657-667. doi:10.1139/ G08-046. PMID:18650955.

Tamura, K., Dudley, J., Nei, M., and Kumar, S. 2007. MEGA4: Molecular Evolutionary Genetics Analysis (MEGA) software version 4.0. Mol. Biol. Evol. 24: 1596-1599. doi:10.1093/ molbev/msm092. PMID:17488738.

Van Ooijen, J., and Voorrips, R. 2001. JoinMap ß 3.0, software for the calculation of genetic linkage maps. Plant Research International, Wageningen, the Netherlands.

Vinatzer, B.A., Zhang, H.B., and Sansavini, S. 1998. Construction and characterization of a bacterial artificial chromosome library of apple. Theor. Appl. Genet. 97: 1183-1190. doi:10.1007/ s001220051008.

Vinatzer, B.A., Patocchi, A., Gianfranceschi, L., Tartarini, S., Zhang, H.B., Gessler, C., and Sansavini, S. 2001. Apple contains receptor-like genes homologous to the Cladosporium fulvum resistance gene family of tomato with a cluster of genes cosegregating with $V f$ apple scab resistance. Mol. Plant-Microbe Interact. 14: 508515. doi:10.1094/MPMI.2001.14.4.508. PMID:11310738.

Vinatzer, B.A., Patocchi, A., Tartarini, S., Gianfranceschi, L., Sansavini, S., and Gessler, C. 2004. Isolation of two microsatellite markers from BAC clones of the $V f$ scab resistance region and molecular characterization of scab-resistant accessions in Malus germplasm. Plant Breed. 123: 321-326. doi:10.1111/j.14390523.2004.00973.x.

Voorrips, R.E. 2002. MapChart: software for the graphical presentation of linkage maps and QTLs. J. Hered. 93: 77-78. doi:10. 1093/jhered/93.1.77. PMID:12011185.

Xu, M., and Korban, S. 2002. A cluster of four receptor-like genes resides in the $V f$ locus that confers resistance to apple scab disease. Genetics, 162: 1995-2006. PMID:12524365. 